\title{
Virtual Synchronous Generator Control of VSC-HVDC System Based on MMC of Hybrid Topology
}

\author{
Jie Wu iD and Chuanjiang $\mathrm{Li}$ (iD \\ Mechanical and Electrical Engineering, Shanghai Normal University, Shanghai 200234, China \\ Correspondence should be addressed to Chuanjiang Li; licj@shnu.edu.cn
}

Received 13 April 2020; Accepted 21 May 2020; Published 17 June 2020

Academic Editor: Zhixiang Zou

Copyright (c) 2020 Jie Wu and Chuanjiang Li. This is an open access article distributed under the Creative Commons Attribution License, which permits unrestricted use, distribution, and reproduction in any medium, provided the original work is properly cited.

\begin{abstract}
For a VSC-HVDC transmission system based on a hybrid topology converter of full-bridge and half-bridge, a kind of virtual synchronous generator (VSG) control strategy which can be applied to modular multilevel converter (MMC) grid-connected structure was researched and proposed. First, based on the conventional VSG control strategy, the energy stored in the equivalent capacitor of MMC power module was used to imitate the rotor inertia of synchronous generator. The characteristics of generator can be simulated during transient frequency fluctuations and it can help relieve the power fluctuations. Secondly, with respect to the structural characteristics of the direct grid connection through the reactor on the AC side of the MMC, which is unlike the microgrid inverter, there are no additional filter capacitors. So, the existing commonly used VSG control strategy of the microgrid inverter and double-closed-loop structure composed of filter capacitor voltage and AC current cannot be directly applied. For this, a method where the given values of inner current loop are calculated by grid impedance matrix was used. So, a double-closed-loop control structure composed of a power outer loop based on VSG control and a current inner loop is obtained. It can effectively improve the current control capability during the transient process. Finally, a hybrid MMC simulation model was built based on PSCAD to verify the correctness and effectiveness of the proposed methods.
\end{abstract}

\section{Introduction}

With the development of microgrid technology and the continuous expansion of new energy grid-connected capacity, the proportion of large-capacity converters represented by VSC-HVDC (Voltage Source Converter Based on High Voltage Direct Current) in the power system is increasing fast. Vector control strategy is usually adopted in existing VSC-HVDC projects. It is easy to achieve independent control of active and reactive power, and dynamic response speed is fast. However, it cannot provide damping and inertial support to the power grid. So, large-scale new energy accessing to the power system is a threat to the stable operation of the power grid. Especially for the weak AC systems, the conventional vector control strategy cannot guarantee the stability of the system.

Large-scale access to the power system will pose a threat to the stable operation of the power grid. Especially when connecting weak AC systems, the conventional vector control strategy cannot guarantee system stability. In this regard, virtual synchronous machine (VSG, virtual synchronous generator) technology came into being. It improves the outer loop control structure of the converter and simulates the characteristics of rotor motion equation and speed regulation of the synchronous generator, based on the synchronous generator control method and operating characteristics in the conventional power system. This can provide inertia and damping for the grid and can improve the stability of VSC-HVDC system for large-scale new energy access [1-4]. The existing VSG technology is widely used in the control of microgrid converters and new energy grid-connected inverters [5]. In [6], the VSG outer ring is designed for distributed energy grid-connected inverters, including two outer control loops of active power and reactive power closed loop. The outputs of the above outer loops are the frequency and amplitude of AC voltage. The 
inner loop is an AC filter capacitor voltage loop and current loop in series. And a small-signal model is established for analyzing system stability. In [7], the stability of VSG control under various fault conditions is analyzed, when the converter is connected to a weak $\mathrm{AC}$ system, indicating that the system can be stabilized as long as the short-circuit ratio is slightly larger than 1 . In [8], the VSG control structure is designed for the module multilevel converter (MMC) based on half-bridge power unit and the stability range of the control parameters is analyzed for accessing weak AC system. In [9], when multiple converters are connected in parallel to the same DC network, there is a low-frequency oscillation risk on the DC side. For this, the weak-damping characteristics of the loops are analyzed based on smallsignal model and an improved VSG control method is proposed to suppress the system oscillation. In $[10,11]$, the VSG control is applied to the microgrid inverter, and the smooth switching method between islanding and gridconnected operation mode is proposed. In the above VSG control strategy, the grid synchronous angular velocity is obtained by the outer loop of active power, and the synchronous angular is calculated by integration. So, the characteristics of the synchronous generator rotor motion equation are obtained, that is, controlling the output power by adjusting the phase difference between the output voltage vectors of the converter valve and the grid. But for the DC voltage control methods are less discussed. However, in fields such as VSC-HVDC, it is necessary to have at least one converter station to stabilize the DC voltage of network. For this, in [12], the DC capacitor energy storage $\left(V_{\mathrm{dc}}^{2} ; V_{\mathrm{dc}}\right.$ is the DC voltage) is used to provide system inertia. The error between capacitor energy storage actual value and the set value is connected with PI regulator, and its output is synchronous angular frequency of the converter voltage vector. In addition, in order to improve the control ability of $\mathrm{AC}$ current, the inner loop is designed, including internal AC voltage closed loop with AC current closed loop in series. It can keep the DC capacitor storage constant and suppresses overcurrent during transients. But in this paper the AC side of the converter is connected to the AC filter capacitor, which is different from the MMC topology of VSC-HVDC projects. The grid-side filter capacitor is mainly used to eliminate the high-order harmonics of the two-level converter output and also stabilizes the grid-connected point voltage, which also provides the necessary conditions for the inner loop design (the inner loop is composed of a filter capacitor voltage closed loop and AC current one in series). For the MMC topology, the AC side is directly connected to the grid via the transformer, no filter capacitor being used, so the inner current loop is difficult to construct. For the direct grid-connected structure of the MMC via the reactance without filter capacitor, in [13, 14], the converter voltage reference signals are calculated by VSG outer loops, and then the reference value of inner current loop is calculated directly by grid-connected impedance of MMC and the voltage difference across it. In [15], the DC voltage and active power reference will change with the frequency deviation. It can improve damping performance of the system and emulate the action of the speed governor of the synchronous generator. However, the grid frequency feedback value is dependent on the phase-locked loop (PLL) in the control structure, where it is difficult to achieve smooth switching between islanding and grid-connected operation modes, and it is useful for VSC-HVDC converters. In [16], it is also proposed that the DC side capacitor energy storage is used to emulate the inertia of the synchronous generator. The DC voltage reference value is changed according to the AC system frequency feedback value and then the conventional double-closed-loop control (DC voltage and current loops) is used. So, it can provide inertia for the frequency changes in the power system. But the inner loop is designed based on PLL and it may affect system stability when the converter is connected to a weak AC system.

With the further development of VSC-HVDC technology and microgrid technology, DC fault clearing capability has become an essential requirement for these kinds of engineering. For this, the Zhangbei VSC-HVDC project, which is DC ring network and has been put into operation in China, adopts DC circuit breaker technology. And the Wudongde project under construction adopts full-bridge-half-bridge hybrid topology to realize the requirement of DC fault crossing. There are few applications of this kind of hybrid topology for engineering, and its control mode is different from the conventional half-bridge topology. The VSG control for this kind of topology is rarely discussed in the literature. In this paper, a VSG control method is proposed for the control structure of this topology, which can increase the inertia of the converter and improve the stability of the system.

\section{Control Method of Hybrid Topology MMC}

The power unit of full-bridge power unit can block bidirectional current, so replacing a certain number of halfbridge units in a traditional half-bridge MMC topology with a full-bridge unit can achieve DC fault clearing with less increasing converter losses. The hybrid MMC is shown in Figure 1.

In the figure, $U_{j}(j=a, b, c)$ is three-phase $\mathrm{AC}$ voltage of valve side, $I_{j}$ is the three-phase AC current, $V_{\mathrm{dc}}$ is the DC side voltage, $I_{\mathrm{dc}}$ is the $\mathrm{DC}$, and $L_{0}$ is the bridge arm reactance. $\mathrm{HB}$ is a full-bridge power module and $\mathrm{FB}$ is a half-bridge one. Each bridge arm is composed of $N_{h}$ half bridges and $N_{f}$ fullbridge power units connected in series. Since only the FB can output a negative voltage, the ratio of the FB mainly affects DC fault clearance and reactive power output capacity of the converter during zero DC voltage operation, etc. Usually the ratio of full-bridge to half-bridge is $50 \%$. In order to achieve fast DC fault clearing and suppress DC short-circuit current rise, the hybrid topology usually sets a DC loop to generate the DC component of the upper and lower bridge modulation signals. When a DC fault is detected (the DC voltage is too low and the DC is overcurrent), the DC component of the modulation signal can be directly set to 0 or a small negative value for DC fault clearing. Meanwhile, the DC voltage outer loop in the conventional VSC-HVDC control structure is usually replaced by the capacitor voltage average control loop of power modules for keeping AC side and DC 


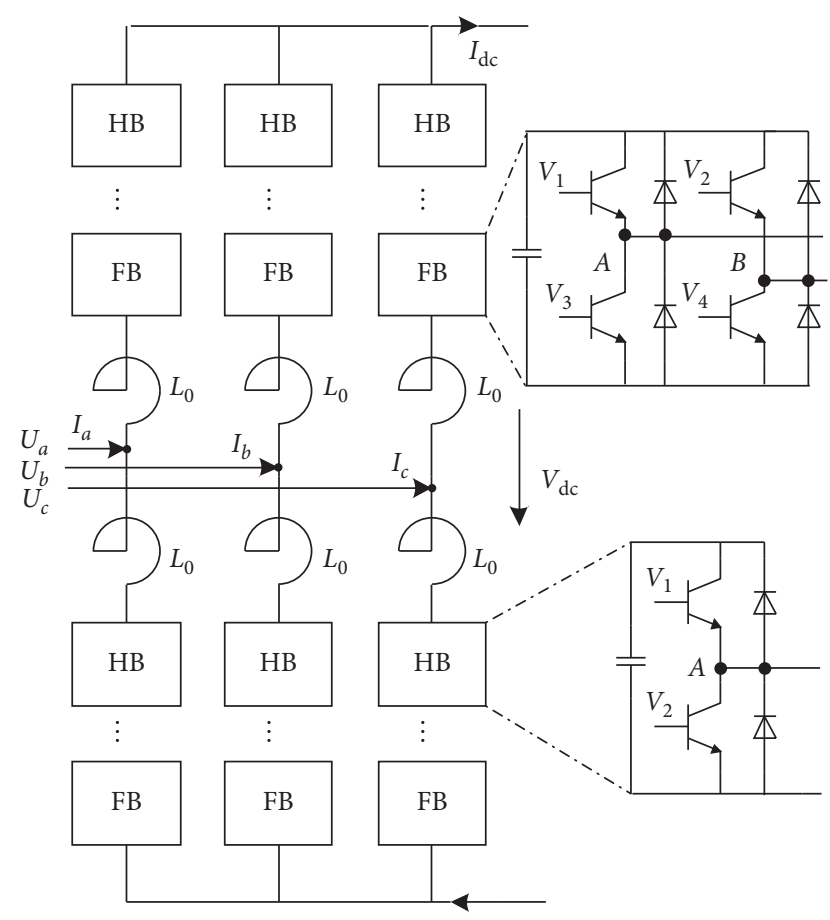

Figure 1: Circuit of hybrid MMC topology.

side power balance [17-19]. The inner current loop is the same as the MMC topology based on HB, and the decoupling control is performed under the synchronous rotation $d q$ frame. The overall control structure is shown in Figure 2.

In the figure, $v_{c_{-} \text {avg }}$ and $v_{c_{-} \text {avg }}^{\text {ref }}$ are the average value of the six bridges arm power unit voltages and the given value. Their error is connected with PI regulator and its output is active current reference value $I_{d \text { ref. }}$. And $I_{\text {qref }}$ is the reactive current reference value. $i_{d}$ and $i_{q}$ are the feedback values of active and reactive current respectively. $I_{\text {dcref }}$ is the DC reference value. The error between $I_{\text {dcref }}$ and its feedback is connected with PI regulator, which outputs the common mode component of the modulation signal $V_{\mathrm{dc} \_ \text {com. }}$. And then it is superimposed with the AC modulation signals of the six bridge arms. That is, it controls the DC to track the given value by directly changing the DC component of the bridge arm output voltage. $V_{c d}$ and $V_{c q}$ are the $d q$-axis components of the modulation signals. $U_{s d}$ and $U_{s q}$ are the $d q$-axis components of the grid voltage, respectively. $V_{j}$ $(j=a, b, c)$ is an AC control signal of three phases. Then it is added to the output signal $V_{\mathrm{dc} \text { com }}$ of the DC regulator, and finally the outputs $V_{j u}, V_{j n}(j=a, b, c)$ are the upper and lower arm control signals, respectively. The three-phase grid voltage $U_{s j}$ is connected with the PLL to obtain the grid synchronization angular velocity $\omega$, which provides synchronous phase for the coordinate transformation ( $d q$ synchronous rotation frame and $a b c$ stationary frame).

\section{VSG Control of Hybrid Topology MMC}

3.1. VSG-Based Average Arm Voltage Control. The synchronous rotational angular velocity in the above control strategy is given by the PLL. When the weak AC system is

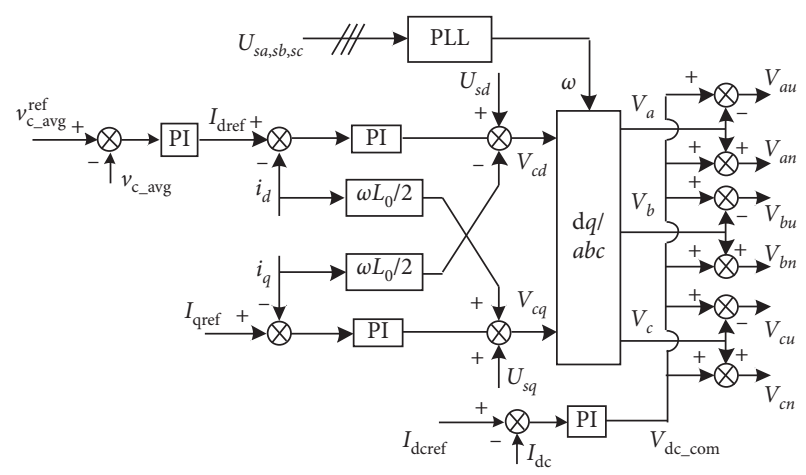

FIGURE 2: Double-closed-loop control structure of hybrid MMC topology.

connected, the phase-locked loop output fluctuates, which may make the system unstable [20]. VSG control can simulate synchronous generator rotor inertia and be used to improve system stability. Rotor motion equation of synchronous generator is shown as follows:

$$
\left\{\begin{array}{l}
2 H \frac{\mathrm{d} \omega}{\mathrm{d} t}=P_{m}-P_{e}-D\left(\omega-\omega_{0}\right), \\
\frac{\mathrm{d} \theta}{\mathrm{d} t}=\omega
\end{array}\right.
$$

In equation (1), $H$ is the inertia time constant, $P_{m}$ and $P_{e}$ are mechanical power and electromagnetic power, $D$ is the damping coefficient, $\omega$ and $\omega_{0}$ are the electrical angular frequency and the rated one, respectively, and $\theta$ is the electrical angle. For the full-half-bridge hybrid topology MMC, the power module capacitor is also an energy storage link, similar to the ability of the synchronous generator rotor to store inertial energy. Therefore, the DC capacitor can be used as a virtual rotor to provide inertia for the system. During steady state, the power on the AC side and the DC side of the converter is equal, and the energy storage of the capacitor is unchanged. It can be expressed by

$$
C V_{c_{-} \text {avg }} \frac{\mathrm{d} V_{c_{-} \text {avg }}}{\mathrm{d} t}=P_{\text {in }}-P_{\text {out }} \text {. }
$$

In the equation, $C$ is the equivalent capacitance of the 6 arms of the converter, $V_{c_{-} \text {avg }}$ is the average value of the capacitor voltage, $P_{\text {in }}$ and $P_{\text {out }}$ are the input and output power of the converter, respectively. From equations (1) and (2), we can obtain

$$
\int\left[2 H \frac{\mathrm{d} \omega}{\mathrm{d} t}+D\left(\omega-\omega_{0}\right)\right]=\int C V_{c_{-} \mathrm{avg}} \frac{\mathrm{d} V_{c_{-} \mathrm{avg}}}{\mathrm{d} t} .
$$

In equation (3), $H$ is the equivalent time constant of the virtual inertia of the converter and $D$ is the damping coefficient. Then, an integral expression for both ends of equation (3) is obtained:

$$
2 H \omega+D \delta=\frac{1}{2} C V_{c}^{2}+K
$$

where $K$ is the integral constant and $\delta$ is the phase of the valve side AC voltage vector. The steady-state value of $\delta$ can be calculated by 


$$
P=\frac{E V}{X} \sin \delta \approx \frac{E V}{X} \delta .
$$

From equation (5), $\delta_{0}$, being the steady-state value of $\delta$, can be expressed as

$$
\delta_{0}=\frac{P_{0} X}{E_{0} V_{0}} .
$$

And the average value of the capacitor voltage is taken as the rated value $V_{c_{-} \text {avg0 }}$. Then, $K$ can be calculated using the steady-state operating point of the system from equation (4):

$$
K=2 H \omega_{0}+D \delta_{0}-\frac{1}{2} C V_{c_{-} a v g 0}^{2} .
$$

Based on the above VSG method and Figure 2, the VSG control structure of the hybrid MMC can be designed as Figure 3.

In the figure, $Q_{\text {ref }}$ and $Q$ are the reference and feedback value of reactive power, respectively. The reference $v_{c_{-} \text {avg }}^{\text {ref }}$ average value of the arm capacitor voltage varies with the system frequency $\omega$ fluctuation, that is, buffering the power fluctuations between AC and DC side of the converter by increasing or reducing the submodule capacitor energy storage and performing AC grid frequency closed-loop control. When the converter output power to the grid is higher than the reference value, the rotation speed of valve side voltage vector increases and the phase difference between the grid voltage and the valve side voltage vector is enlarged. Therefore, the output of frequency controller of AC voltage vector increases. That is, the reference value of submodule capacitance voltage increases and the energy storage increases. The transient power is buffered by the capacitors of submodules and fluctuation of power and angular frequency is reduced. This is equivalent to the inertia and damping characteristics of the synchronous generator rotor, which enables the frequency support of the converter to the grid. For the selection of parameters $H$ and $D$, it is mainly affected by the allowable range of system frequency fluctuations, maybe $\pm 5 \%$. Another limitation is that the calculated reference value of submodule average voltage is within the allowable fluctuation range of the converter.

3.2. VSG Control of Active Power. For the VSC-HVDC system based on hybrid topology, the converter station for constant power control is also directly connected to the grid, without filter capacitor of AC side. So the conventional VSG control strategy for the microgrid inverter cannot be used directly. And, for the hybrid MMC topology, if the outer control loop is designed with conventional VSG strategy, it is necessary to consider how to obtain the reference value of inner current loop. Performing a Laplace transform on equation (1) yields

$$
\left\{\begin{array}{l}
\omega-\omega_{0}=\frac{P_{m}-P_{e}}{2 H s+D}, \\
\theta=\frac{\omega}{s},
\end{array}\right.
$$

where $s$ is differential operator. From equation (8), based on swing equation of synchronous machine, the grid synchronization angular frequency can be obtained. Then, the voltage amplitude of valve side can be obtained by reactivevoltage droop control structure:

$$
V_{\mathrm{ac}}^{\mathrm{ref}}-V_{\mathrm{ac}}=K_{\mathrm{Q}}\left(Q_{\mathrm{ref}}-Q\right) \text {. }
$$

In the equation, $V_{a c}^{r e f}$ and $V_{a c}$ are the amplitude reference and feedback value of valve side $\mathrm{AC}$ voltage, respectively. $K_{Q}$ is the reactive-voltage droop coefficient. From the gridconnected structure of hybrid MMC, the relationship between AC voltage and current of valve side is obtained as shown in equation (10) based on the synchronous rotation $d q$ frame:

$$
L_{0} \frac{\mathrm{d}}{\mathrm{d} t}\left[\begin{array}{l}
i_{c d} \\
i_{c q}
\end{array}\right]=\left[\begin{array}{cc}
R_{L} & -\omega_{0} L_{0} \\
\omega_{0} L_{0} & R_{L}
\end{array}\right] \cdot\left[\begin{array}{l}
i_{d} \\
i_{q}
\end{array}\right]=\left[\begin{array}{l}
U_{s d}-V_{c d} \\
U_{s q}-V_{c q}
\end{array}\right] .
$$

In equation (10), $R_{L}$ is the equivalent resistance of reactor of valve side. From it, the AC reference value can be calculated by grid impedance matrix, which is composed of the reactance and resistance of the MMC AC side:

$$
\left[\begin{array}{c}
i_{d}^{\mathrm{ref}} \\
i_{q}^{\mathrm{ref}}
\end{array}\right]=\left[\begin{array}{cc}
R_{L} & -\omega_{0} L_{0} \\
\omega_{0} L_{0} & R_{L}
\end{array}\right]^{-1} \cdot\left[\begin{array}{c}
U_{s d}-V_{c d} \\
U_{s q}-V_{c q}
\end{array}\right]
$$

The control structure of active and reactive power is shown in Figure 4 based on VSG strategy.

In Figure 4, the reference value of inner current loop is calculated based on the grid-connected impedance matrix. For the problem of strong coupling of the $d q$-axis control loop when connecting weak AC systems, the decoupling control method proposed in [11] can be used to optimize the dynamic performance. The parameters $H$ and $D$ can be selected by the active power closed-loop transfer function. Firstly, according to the second-order system, the appropriate damping ratio and control bandwidth are set for calculation of $H$ and $D$ and then determined by electromagnetic transient simulation. A detailed calculation process $[8,11]$ has been given in many literatures and will not be described again.

\section{Simulation and Analysis}

In order to verify the above control method, a full-halfbridge hybrid MMC model is built based on PSCAD. One terminal takes the active power as the control target, and the control structure is shown in Figure 4. The other one takes the average value of the arm capacitor voltage as the control target, and the control structure is shown in Figure 3. The control structures at both ends can be switched between each other: one end is set to a constant power control mode, and the other end is a constant voltage control mode. It can use this to maintain system power balance. Logic switches can be used to achieve smooth switching between the two outer loop control structures and control objectives [21]. To reduce the amount of calculation, each arm consists of 10 power units connected in series, including 5 half-bridge units and 5 full-bridge ones. The AC system voltage is $10 \mathrm{kV}$ 


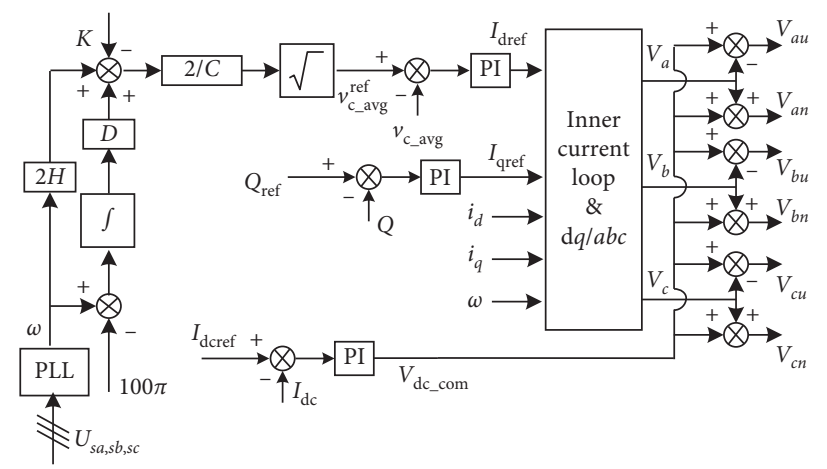

FIGURE 3: Constant capacitor voltage sum control structure based on VSG strategy.

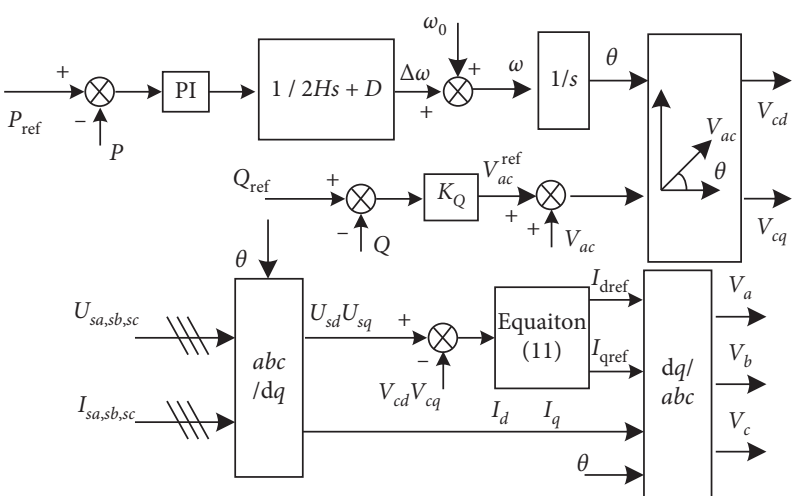

Figure 4: Constant power control structure based on VSG strategy.

and DC rated voltage $20 \mathrm{kV}$, and rated power $30 \mathrm{MW}$. The arm reactance is $5 \mathrm{mH}$. The equivalent inductance of the AC system is set to $5.3 \mathrm{mH}$, and the short-circuit capacity of the system is about $60 \mathrm{MW}$. So it is a weak AC system. It can be verified that the VSG control algorithm can improve the stability of the converter connected to the weak AC system.

System parameters are normalized with peaks of AC voltage and $\mathrm{AC}$ in the simulation. Active and reactive power is normalized by system rated capacity. And adjusted by simulation, $J=0.0471$ p.u. and $D=1.1$ p.u. At $t=4 \mathrm{~s}$, active power step changes from $0 \mathrm{MW}$ to $30 \mathrm{MW}$. The simulation results are shown in Figure 5. The variable label " 1 " in the figure is the output waveform of the conventional doubleclosed-loop control method (Figure 2) for hybrid topology, and the label " 2 " is the output waveform based on VSG strategy. Figures 5(a) and 5(b) are the waveforms of DC voltage and DC, respectively. As shown in the figure, with conventional control strategy, there are large oscillations when the converter is unlocked and during the power step rising. The oscillation attenuation is slow, so the system characteristic is of weak damping. Figure 5(c) is the step response of active power. It can be seen from the control method shown in Figure 4 that the output of the active power regulator is connected to the virtual inertia and damping part $1 /(2 \mathrm{Hs}+\mathrm{D})$ to obtain the synchronization angular frequency adjustment $\Delta w$, that is, by adjusting the phase of the AC output voltage of the converter side to track the active power reference. Because of the existence of inertia part, the power rises slowly during the step, but it can eliminate the power oscillation of the conventional control strategy. In this figure, the output power fluctuates greatly at the unlocking time. The reason is that, in order to simplify the system simulation model, the special presynchronization processing logic is not performed for the initial grid connection time. For the presynchronization process, there have been related literatures $[8,9]$. This is beyond the scope of this paper. The unlocking impact here can be easily solved by setting the relevant presynchronization logic in the practical project [9, 19]. Figure 5(d) shows the phase-locked loop (PLL) output $w 1$ of the conventional control strategy and the grid synchronization angular frequency $w 2$ calculated by the power loop of the VSG control strategy. It can be seen from the simulation results that the PLL output fluctuations during the unlocking and step response are consistent with the oscillation characteristics of the power curve, indicating that the PLL characteristics deteriorate during the transient process, causing the system oscillation, if the weak AC system connected. Figure 5(e) shows the AC voltage vector angular frequency increment $\Delta \omega$ and phase (angle) of valve side, which are the outputs of VSG control part. The power adjustment process is similar to the characteristics of a synchronous generator. When the power command is increased, the generator will increase the output of the prime mover, and then the rotor will accelerate. For the converter, the rotation speed of valve side voltage vector will be increased. It increases the phase difference between the valve side and the grid-side voltage, thereby adjusting the output power of the converter. Figure 5(f) shows the $d q$-axis current 


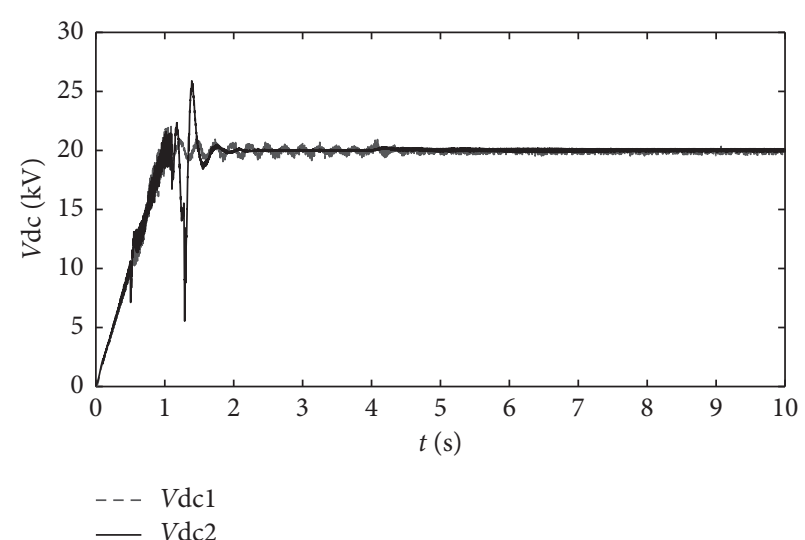

(a)

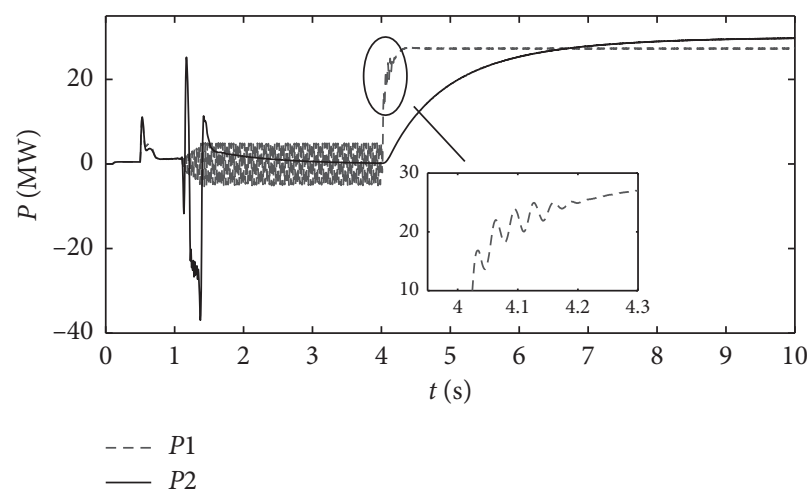

(c)

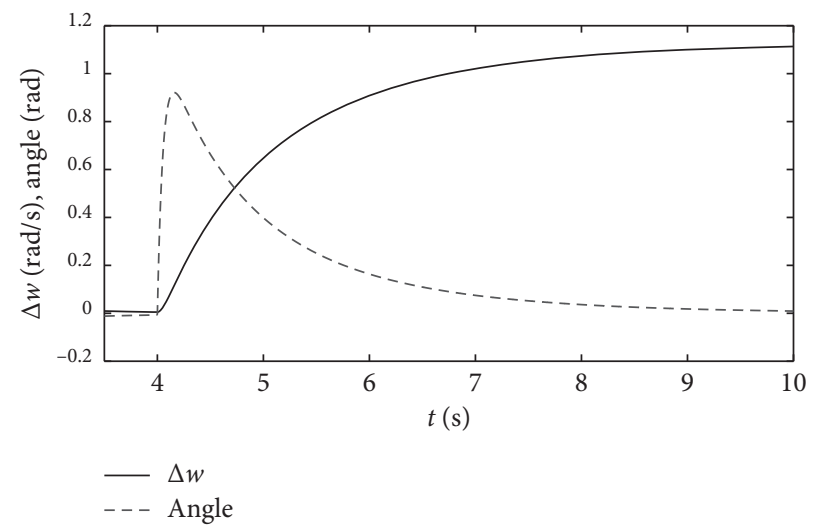

(e)

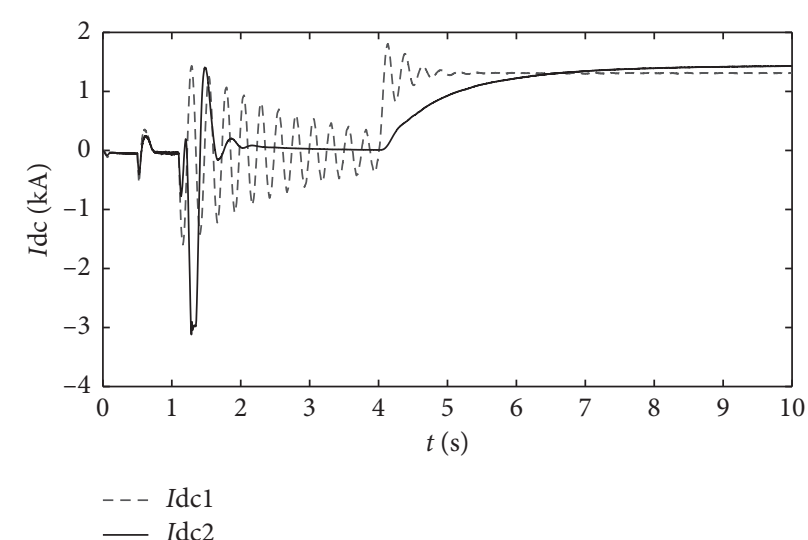

(b)

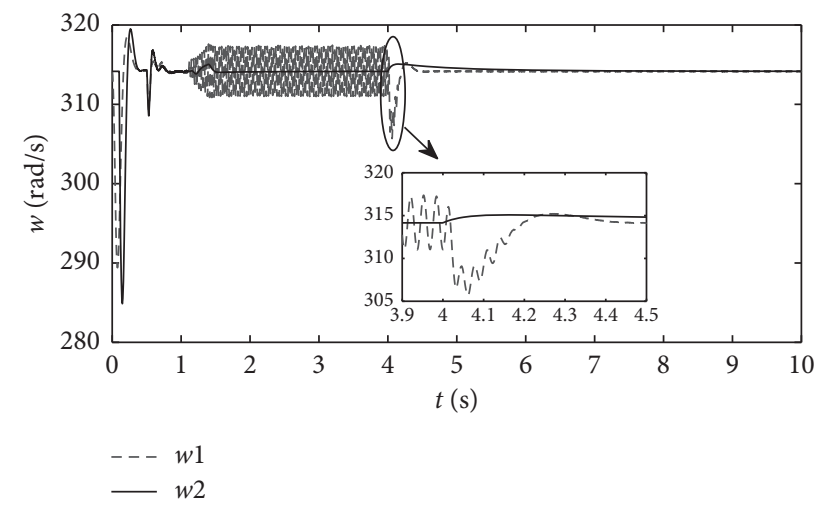

(d)

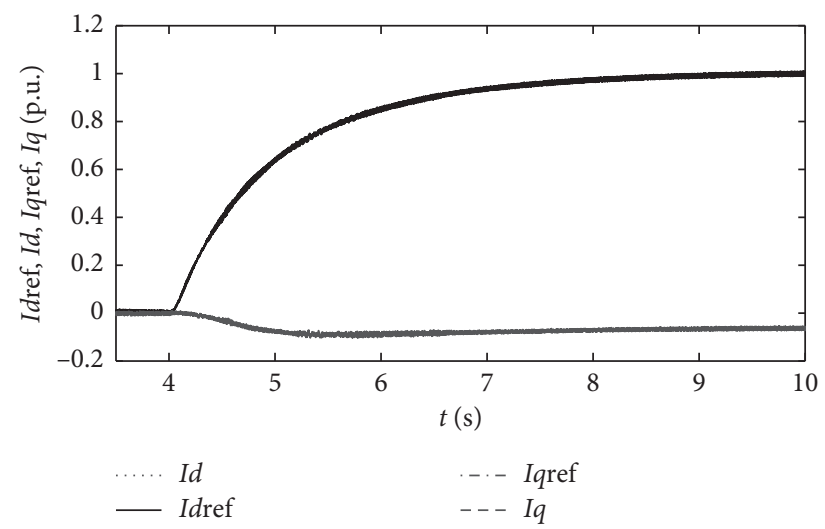

(f)

FIGURE 5: Active power step response by conventional dual closed-loop control and VSG control used for hybrid topology. (a) Waveforms of DC voltage. (b) Waveforms of DC. (c) Waveforms of active power. (d) Grid synchronization angular velocity calculated by PLL and active power controller based on VSG. (e) Difference of synchronized angular velocity between grid and output of VSG control module and phase of valve side voltage. (f) Tracking the reference values of $d q$-axis inner current loop.

reference and feedback values during the step process based on VSG control strategy, and both d-and $q$-axis currents can track the given values during steady-state and step processes.

Hybrid topology MMC can suppress DC short-circuit current. For verification, DC bipolar short-circuit fault is set as $t=8 \mathrm{~s}$. The fault is cleared after $100 \mathrm{~ms}$, and the simulation result is shown in Figure 6. And Figure 6(a) shows the DC voltage and current waveform during the DC fault. Figure $6(\mathrm{~b})$ shows the $d q$-axis current reference and feedback, and there is a small fluctuation of feedback currents during the short-circuit process. Figure 6(c) shows the sum of the capacitor voltages of the three lower arms in the converter $\left(V_{c a n}, V_{c b n}\right.$, and $\left.V_{c c n}\right)$. The upper arm waveforms are symmetrical with the lower ones. Based on the control strategy shown in Figure 3, the increase or decrease of the storage energy of the module equivalent capacitance is used to buffer the system energy in the transient process, and it is used for emulating the inertia of the generator. So, during 


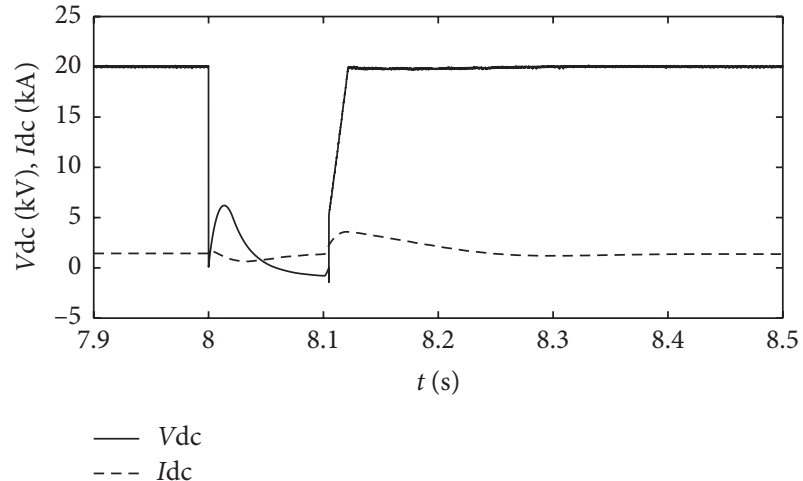

(a)

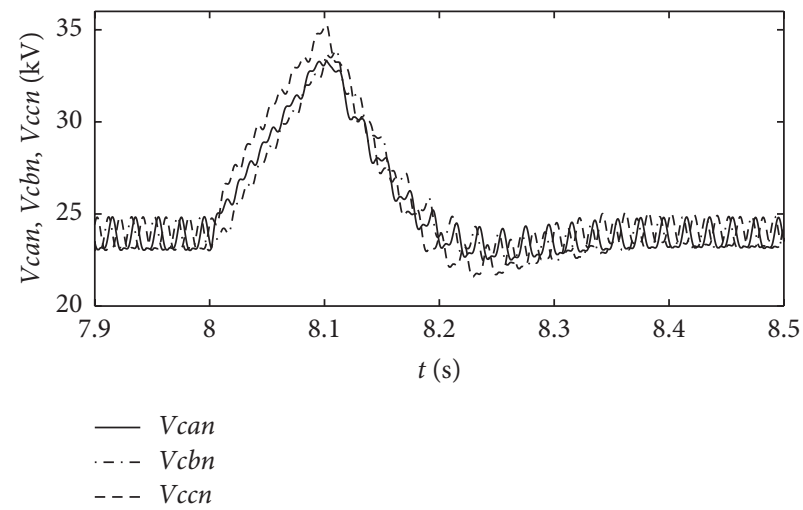

(c)

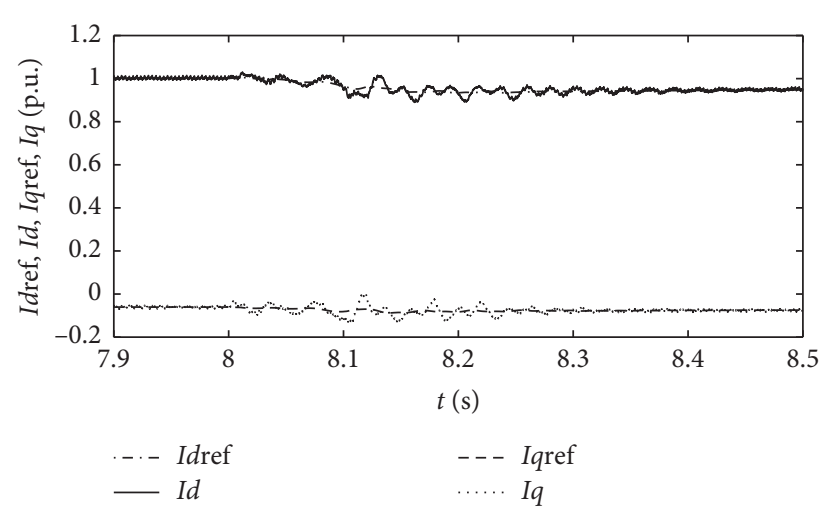

(b)

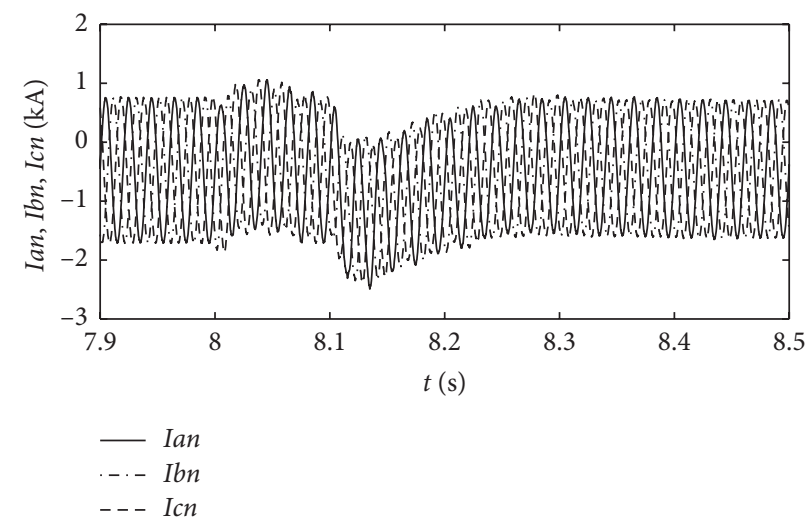

(d)

FiguRE 6: DC short-circuit fault simulation waveform. (a) Waveforms of DC voltage and current. (b) Reference and feedback of inner $d q$ axis current loop. (c) Sum of arm capacitor voltages of converter lower arms. (d) Lower arm current of converter.

the transient process, the sum of arm capacitor voltages rises to buffer the energy. Figure 6(d) shows the three-phase current $\left(I_{a n}, I_{b n}\right.$, and $\left.I_{c n}\right)$ of lower arms of the converter. During DC fault, the output of DC control module rapidly reduces, which is the $\mathrm{DC}$ component of the modulation signals. This can make the DC voltage close to 0 , suppress the DC short-circuit current from rising, and realize the transient DC fault clearing.

\section{Conclusion}

First of all, VSC-HVDC system based on VSG control can emulate synchronous generator characteristics. Capacitor voltage average of arms and active power can be controlled by adjusting the angular frequency of voltage vector of converter side. The grid synchronization angle is generated by the VSG control loop. It can eliminate the system oscillation caused by the PLL during transient and effectively improve system stability, when the converter valve is connected to the weak AC system. Second, PLL is needed for VSG strategy to track grid phase before it is connected to the grid. The control system needs to perform the presynchronization processing of the output voltage amplitude and the phase with grid. Otherwise, the grid connection will cause a large current surge. Finally, for DC faults, the DC output voltage of hybrid topology MMC can be reduced quickly by using the DC control part and the short-circuit current rising can be suppressed. In summary, VSG control technology applied to hybrid topology MMC can effectively improve the DC access capability of weak AC systems and clear DC fault. And there is broad application prospect in the fields of VSC-HVDC system, new energy access, microgrid, and so on.

\section{Data Availability}

The data used to support the findings of this study are included within the article.

\section{Conflicts of Interest}

The authors declare that they have no conflicts of interest.

\section{Acknowledgments}

This work was supported by the National Science Foundation (Grant no. 61801293).

\section{References}

[1] H.-P. Beck and R. Hesse, "Virtual synchronous machine," in Proceedings of the 9th International Conference on Electrical Power Quality and Utilisation, Barcelona, Spain, October 2007. 
[2] G. Yao, Z. Lu, B. Mohamed et al., "A virtual synchronous generator based inverter control method for distributed generation systems," in Proceedings of the IECON 2015-41st Annual Conference of the IEEE Industrial Electronics Society, Yokohama, Japan, November 2015.

[3] T. Zheng, L. Chen, T. Chen et al., "Review and prospect of virtual synchronous generator technologie," Automation of Electric Power System, vol. 39, no. 27, pp. 165-175, 2015.

[4] L. Zhu, Z. Yuan, S. Chao et al., "Review of frequency support control methods for asynchronous interconnection system based on VSC-HVDC," Electric Power Automation Equipment, vol. 39, no. 2, pp. 84-92, 2019.

[5] J. Meng, Y. Wang, X. Shi et al., "Control strategy and parameter analysis of distributed inverters based on VSG," Transactions of China Electro Technical Society, vol. 29, no. 12, pp. 1-10, 2014.

[6] S. Lu and X. U. Zheng, "Analysis of the maximum power flow in power synchronization control based MMC-HVDC," Proceedings of the CSEE, vol. 36, no. 7, pp. 1868-1876, 2016.

[7] W. Liu, C. Guo, C. Zhao et al., "Power damping synchronization control for MMC-HVDC connected to an extremely weak receiving AC grid," Proceedings of the CSEE, vol. 36, no. 17, pp. 4647-4656, 2016.

[8] H. Yuan, X. Yuan, and J. Hu, "Modeling of grid-connected VSCs for power system small-signal stability analysis in DClink voltage control timescale," IEEE Transactions on Power Systems, vol. 32, no. 5, pp. 3981-3991, 2017.

[9] L. Ü Zhipeng, W. Sheng, Q. Zhong et al., "Virtual synchronous generator and its applications in micro-grid," Proceedings of the CSEE, vol. 34, no. 16, pp. 2591-2603, 2014.

[10] R. Shi, X. Zhang, F. Liu et al., "Control technologies of multienergy complementary microgrid operation based on virtual synchronous generator," Transactions of China Electro Technical Society, vol. 31, no. 20, pp. 170-180, 2016.

[11] L. Huang, H. Xin, Z. Wang et al., "A virtual synchronous control for voltage-source converters utilizing dynamics of DC-link capacitor to realize self-synchronization," IEEE Journal of Emerging and Selected Topics in Power Electronics, vol. 5, no. 4, pp. 1565-1577, 2017.

[12] M. Li, Y. Wang, N. Xu et al., "A novel virtual synchronous generator control strategy based on improved swing equation emulating and power decoupling method," in Proceedings of the IEEE Energy Conversion Congress and Exposition, Milwaukee, WI, USA, September 2016.

[13] B. Gao, C. Xia, L. Zhang et al., "Modeling and parameters design for rectifier side of VSC-hvdc based on virtual synchronous machine technology," Proceedings of the CSEE, vol. 37, no. 2, pp. 534-544, 2017.

[14] Y. Cao, W. Wang, Y. Li et al., "A virtual synchronous generator control strategy for VSC-MTDC system," IEEE Transactions on Energy Conversion, vol. 33, 2018.

[15] J. Zhu, C. D. Booth, G. P. Adam, A. J. Roscoe, and C. G. Bright, "Inertia emulation control strategy for VSC-hvdc transmission systems," IEEE Transactions on Power Systems, vol. 28, no. 2, pp. 1277-1287, 2013.

[16] S. Li, X. Wang, T. Li et al., "Optimal design for hybrid MMC and its DC fault ride-through strategy," Proceedings of the CSEE, vol. 36, no. 7, pp. 1849-1858, 2016.

[17] W. Lin, D. Jovcic, S. Nguefeu, and H. Saad, "Full-bridge MMC converter optimal design to HVDC operational requirements," IEEE Transactions on Power Delivery, vol. 31, no. 3, pp. 1342-1350, 2016.

[18] G. P. Adam and I. E. Davidson, "Robust and generic control of full-bridge modular multilevel converter high-voltage DC transmission systems," IEEE Transactions on Power Delivery, vol. 30, no. 6, pp. 2468-2476, 2015.

[19] L. Zhang, H.-P. Nee, and L. Harnefors, "Analysis of stability limitations of a VSC-hvdc link using power-synchronization control," IEEE Transactions on Power Systems, vol. 26, no. 3, pp. 1326-1337, 2011.

[20] W. E. I. Yalong, H. Zhang, K. Sun et al., "Pre-synchronization method of virtual synchronous generator using virtual power," Automation of Electric Power System, vol. 40, no. 12, pp. 124-129, 2016.

[21] J. Wu and Z. Wang, "Improved droop control strategy for multi-terminal voltage Source converter-HVDC," Transaction of China Electrotechnical Society, vol. 32, no. 20, pp. 241-250, 2017. 